ticated exposition of the research process, its relation to theory and, in this case where time was not pressing, for the demonstration that the amount of thought and expertise invested in such research has its intellectual pay-off and its social uses. The other government-initiated study, the Survey of Income and Program Participation (in social welfare programmes), gives Hunt occasion to trace the history of survey research and indicate what is considered to be current best practice.

A fourth case takes the example of a social psychological laboratory experiment with undergraduates to illustrate the great power of this method, which dominates American social psychology, and its inherent problems: Do the results apply to the world outside the laboratory? Is the desire for experimental precision responsible for all too many elegant trivialities in the literature? A final case exemplifies the methods of longitudinal panel research through analysis of some studies, designed to continue for 20 years, of aging in an originally healthy middle-aged sample.

Altogether this is an engagingly and clearly written book. It presents social science as it is, warts and all. Social scientists could well use it as a textbook. Natural scientists may gain from it insight into the intellectual challenges social scientists face, and respect for their efforts in meeting them.

Marie Jahoda is Emeritus Professor of Social Psychology at the University of Sussex, Falmer, Brighton BN1 $9 Q G, U K$.

\section{Inflamed appeal}

\author{
Gerry Higgs \& Brendan Whittle
}

The Inflammatory Reaction. By Henry Z. Movat. Elsevier:1985. Pp.365. Dfl.260.

THE inflammatory reaction continues to be a centre of interest in the medical sciences, largely because of the high incidence of inflammatory diseases throughout the world. One in three people in the West can expect to suffer from some sort of chronic inflammatory disorder during the course of their life. This book, however, is devoted essentially to the acute inflammatory reaction and here Professor Movat has dealt very thoroughly with the subject. He makes no apology for his somewhat idiosyncratic approach, drawing heavily upon his own research findings to illustrate and explain the events of inflammation. This is not to say that the book lacks objectivity. It is an impressive review of current knowledge, with an extensive bibliography that takes up more than a fifth of the text.

The study of inflammation was for many years considered the province of pathologists and has only recently been hijacked by pharmacologists and immunologists. Professor Movat emphasizes the need for integration of the disciplines concerned, but, as a pathologist himself, he is well placed to lead his readers through the historical developments and on to current ideas. Not surprisingly then, he places inflammation in the context of the structure and function of the tissues affected. Beginning with the anatomy of the microcirculation, he goes on to describe the vascular and lymphatic changes occurring as a result of inflammatory injury. The morphological and functional aspects of leukocyte activation are dealt with in a similar way. This approach provides a good basis for the chapters on mediators and mechanisms.
The longest chapter, occupying almost a quarter of the book, tackles the formidable list of mediators that are known to contribute to the vascular phenomena of acute inflammation. Another chapter deals with substances which stimulate leukocyte emigration. The problem with categorizing inflammatory mediators is that they are rarely involved in discrete functions. For example, the prostaglandins, leukotrienes and complement-derived factors contribute to vascular as well as cellular responses in inflammation. Professor Movat has overcome this difficulty by demonstrating the relative importance of individual mediators in processes such as vasodilation, vascular permeability, chemotaxis and degranulation. This has not been achieved without cross-referencing between chapters, which in places is confusing. The picture that emerges, however, is that inflammatory reactions are initiated and amplified by the actions and synergistic interactions of a large number of mediators.

It is a little disappointing that the mechanisms of chronic inflammation are not touched upon until the penultimate chapter of the book. Also, discussions of the treatment of inflammation or the mechanisms of anti-inflammatory drugs have been deliberately avoided. Nevertheless, this is the most comprehensive and up-to-date text available on the subject of acute inflammation, and as such it will prove useful for reference. A large part of the book is based on experimental findings and this aspect should appeal to the post-graduate and post-doctoral workers in inflammation research. Professor Movat should, therefore, succeed in his objective of stimulating the young investigators to whom this monograph is primarily addressed.

Gerry Higgs is Head of the Anti-Inflammatory partment of Mediator Pharmacology at the Wellcome Research Laboratories, Langley Court, Beckenham, Kent BR3 $3 B S, U K$. Section and Brendan Whittle Head of the De-

\section{Engineered viruses out of doors}

\author{
D.H.L. Bishop
}

Genetically Altered Viruses and the Environment. Edited by Bernard Fields, Malcolm A. Martin and Daphne Kamely. Cold Spring Harbor Laboratory: 1986. Pp.362. \$63.

THE use of genetically altered viruses in medicine or veterinary practice is, of course, old hat. Since the late eighteenth century and Jenner's pioneering work on smallpox, attenuated genetic variants of disease-causing viruses have been employed to protect man and domestic animals from infection by virulent viruses.

Such vaccine strains of viruses have been obtained by serendipity. What then is the concern about genetically altered viruses in the environment? With modern technologies and precise knowledge of the genetics of viruses (genome sequences and coding strategies), not only can we now achieve the objectives of earlier scientists by direct and specific genetic engineering, but we can go much further in custom-designing viruses for new purposes. It is against this background that the symposium that spawned this book was organized. The issues raised are both broad and far-reaching, and are of importance not only to scientists but also to the public, especially local and national regulatory authorities.

The initial chapters of the book describe the US legislative and regulatory frameworks pertaining to the use of genetically engineered viruses. Corresponding frameworks in other countries are not considered, limiting the interest of this information to the international audience, though one appreciates from these accounts the problems that have arisen in the United States where regulations are being applied to a subject that goes beyond the concepts involved in originally formulating the legislation.

The development and use of genetically engineered viruses in agriculture and forestry will open up new tracts of science, and will doubtless have irreversible environmental consequences. The question of risk assessment is therefore a central one, but there is only restricted discussion of this subject in the book. It was, for example, a major oversight of the organizers that there is a detailed report neither of the use in Australia of the South American myxomatosis virus to control rabbit populations, nor of the domino effects of the introduction of the virus to continental Europe and the United Kingdom. Likewise our extensive knowledge of the use of viral insecticides to control pests of agriculture and forestry is given 